Wilfrid Laurier University

Scholars Commons @ Laurier

Physics and Computer Science Faculty

Publications

Physics and Computer Science

$11-1988$

\title{
Fabrication of Tapers and Lenslike Waveguides by a Microcontrolled Dip Coating Procedure
}

Daniel W. Hewak

University of Waterloo

John W.Y. Lit

Wilfrid Laurier University, jlit@wlu.ca

Follow this and additional works at: https://scholars.wlu.ca/phys_faculty

\section{Recommended Citation}

Hewak, Daniel W. and Lit, John W.Y., "Fabrication of Tapers and Lenslike Waveguides by a Microcontrolled Dip Coating Procedure" (1988). Physics and Computer Science Faculty Publications. 11.

https://scholars.wlu.ca/phys_faculty/11

This Article is brought to you for free and open access by the Physics and Computer Science at Scholars Commons @ Laurier. It has been accepted for inclusion in Physics and Computer Science Faculty Publications by an authorized administrator of Scholars Commons @ Laurier. For more information, please contact scholarscommons@wlu.ca. 


\title{
Fabrication of tapers and lenslike waveguides by a microcontrolled dip coating procedure
}

\author{
Daniel W. Hewak and John W. Y. Lit
}

\begin{abstract}
A technique for the fabrication of tapered and lenslike waveguides from solution-deposited thin films is described. Using a microprocessor controlled dipping arm, substrates are withdrawn from a solution with a carefully controlled and varying velocity. In this way optical waveguides with regions of varying thickness are deposited. Following the drying and baking of the films, desired structures are obtained in hard inorganic optical waveguides of good optical quality. With refined profile control, we propose to fabricate other optical waveguide components, for example, thin film lenses with this method.
\end{abstract}

\section{Introduction}

For many years, solution deposition has been studied as a possible way to make planar optical waveguides. ${ }^{1-3}$ Recently there has been renewed interest in this method for guided wave or integrated optics applications. Low-loss high-quality waveguides have been fabricated, ${ }^{3-6}$ and an embossing technique has been described for obtaining integrated optical components such as surface-relief gratings. ${ }^{4-6}$ Here we report on the fabrication of thickness varying waveguide structures directly from solution, without embossing, by a microprocessor controlled dip coating procedure. We have used this technique to fabricate tapered optical waveguides and lenslike waveguides from a commercially available $\mathrm{SiO}_{2}: \mathrm{TiO}_{2}$ solution. The solution (trade name Liquicoat and available from E. Merck) contains a silic acid ester and titanic acid ester in a stabilized colloidal solution. By varying the ratio of the two components, the refractive index of the resulting films can be adjusted from $\sim 1.4$ to 2.0. A singledip coating typically yields a thin film structure 0.05 $0.25 \mu \mathrm{m}$ thick.

Daniel Hewak is with University of Waterloo, Guelph-Waterloo Program for Graduate Work in Physics, Waterloo, Ontario N2L $3 G 1$, Canada, and John Lit is with Wilfrid Laurier University, Department of Physics \& Computing, Waterloo Ontario N2L 3C5, Canada.

Received 20 January 1988.

0003-6935/88/214562-03\$02.00/0.

(C) 1988 Optical Society of America.

\section{Solution Deposited Planar Waveguides}

The details of a typical dip coating procedure have been described elsewhere. ${ }^{5-7}$ Briefly, substrates are lowered into the solution and withdrawn vertically with a smooth and constant velocity. The films are dried while in a vertical position for a few minutes at $\sim 100^{\circ} \mathrm{C}$ and then baked at a high temperature, usually up to the strain point of the substrate, to harden the film and drive off any solvents remaining in the waveguiding material.

The resulting waveguides have proved to be of good optical quality, being hard, resistant, and with low losses. These optical properties have been previously discussed in detail. Briefly, Tienfenthaler et al. ${ }^{5}$ measured losses both with a light scattering method and by examining the attenuation between prism incoupling and outcoupling. Losses varied from 0.21 to $6.61 \mathrm{~dB} /$ $\mathrm{cm}$ depending on the wavelength, $\mathrm{TiO}_{2}, \mathrm{TiO}_{2}$ content, and the method of measurement. Herrman and Wild$\mathrm{mann}^{3}$ studied the losses, optical damage thresholds, and chemical and mechanical durability. Losses were $<1.12 \mathrm{~dB} / \mathrm{cm}$, and the films withstood pulsed energy of $142 \mu \mathrm{J}$ at $514 \mathrm{~nm}$, coupled into a $\mathrm{TE}_{0}$ mode. Repeated cleaning, handling, and measurement demonstrated that the films are very durable and stable over long time periods (up to $1 \mathrm{yr}$ ).

\section{Thickness Varying Structures}

To obtain waveguides with regions of varying thickness, we have modified this dip coating procedure by providing a microprocessor controlled dipping arm to lift the substrate from the solution with a predetermined varying velocity. The thickness of the thin film varies directly with the velocity of the lifting arm; thus by varying the rate of withdrawal from the solution, the thickness of the resulting waveguide can be con- 
a.
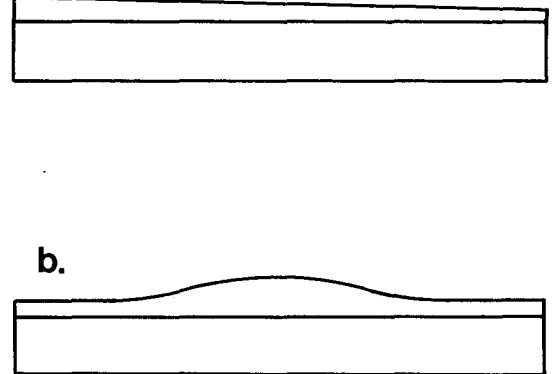

Fig. 1. Typical structures fabricated with the microprocessor controlled dip coating procedure: (a) tapered optical waveguide and (b) lenslike waveguide.

trolled. Two properties of the dip coating solution allow the fabrication of practical structures. First, the solvent used in the solution has a very high rate of evaporation allowing the solution-deposited film to immobilize almost instantly on the substrate on withdrawal. Consequently, at low dipping speeds, there is no evidence of flow lines or irregularities in the film, and all thickness changes are linear and perpendicular to the direction of withdrawal. Second, the solution is of low viscosity; thus despite the high rate of evaporation, all thickness changes are smooth and continuous. After the microprocessor controlled dip coating, the resulting thin films are dried and baked as for a planar waveguide. With this procedure we have fabricated tapered waveguides and lenslike waveguides, as illustrated in Fig. 1.

To achieve a desired thickness profile in a predictable and reproducible manner an understanding of the effects of many variables which influence the final film thickness and refractive index is required. These variables include the dipping speed, the temperature, and duration of drying and baking, and the solution viscosity, which varies with temperature, and the $\mathrm{SiO}_{2}: \mathrm{TiO}_{2}$ ratio. To standardize the dip coating procedure and allow the calibration of the parameters which determine the final thin film properties, we have extensively examined the dependence of film thickness and refractive index on these and other variables. Details of this study are presented elsewhere. ${ }^{7}$ However, the more important and relevant results are shown in Figs. 2 and 3. All dip coating was done with the solution and substrate at room temperature. We have used glass microscope slides and Corning 7059 glass $(75 \times 25 \times 1$ $\mathrm{mm}$ ) as substrates. Dip coating deposits a film on both sides of the substrate. However, prior to baking, the unwanted film could be removed by wiping with pure acetone and a lens cleaning tissue. Measurements of thickness and refractive index were made using ellipsometry at wavelength $\lambda=0.633 \mu \mathrm{m}$ and confirmed with a surface profiler.

Figure 2 shows the thicknesses achievable with a single dip coating. Films shrink by $\sim 50 \%$ of their original thickness during baking, yielding final thicknesses of $0.05-0.25 \mu \mathrm{m}$, which corresponds to a singlemode waveguide. Film shrinkage varies slightly with

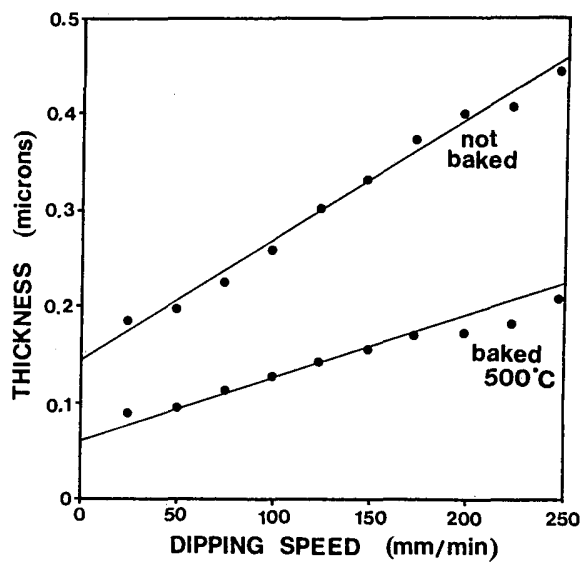

Fig. 2. Film thickness as a function of dipping speed before baking and after baking at $500^{\circ} \mathrm{C}$ for $1 \mathrm{~h}$.

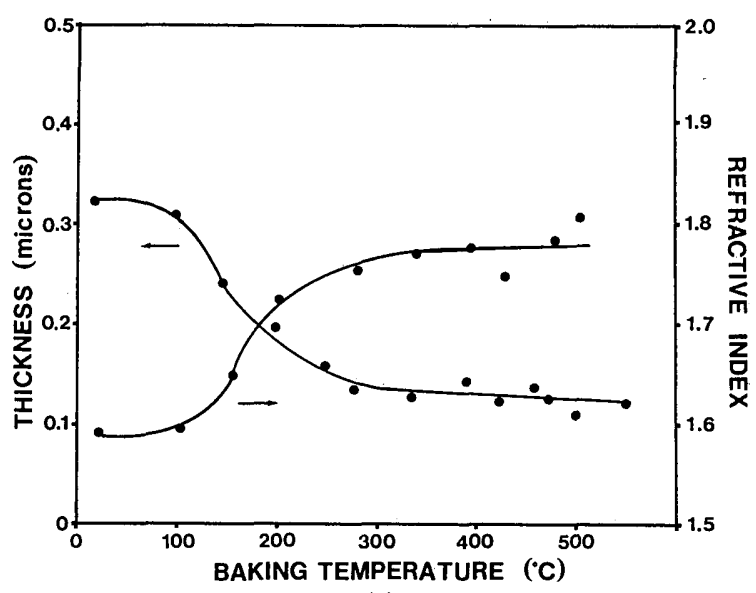

(a)

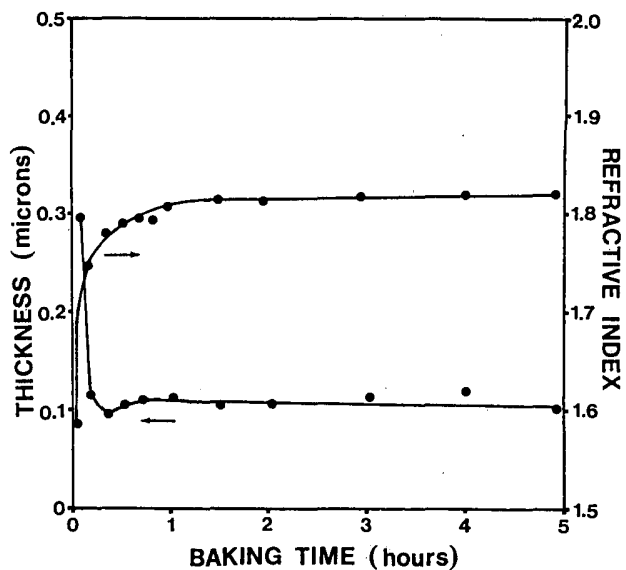

(b)

Fig. 3. (a) Effects of baking temperature and (b) baking time on film thickness and refractive index for films withdrawn at $72.4 \mathrm{~mm} /$ $\min$.

initial thickness with the relative shrinkage of thicker films slightly less.

The effects of drying time and temperature on the final thin film properties are small. However, the effects of baking temperature and time, as shown in Fig. 3, must be considered. Low baking temperatures 


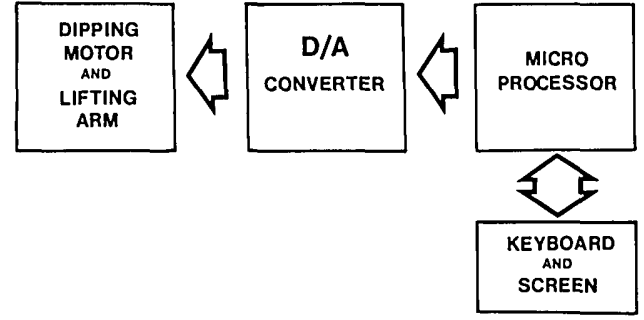

Fig. 4. Schematic of the microprocessor controlled dip coating.

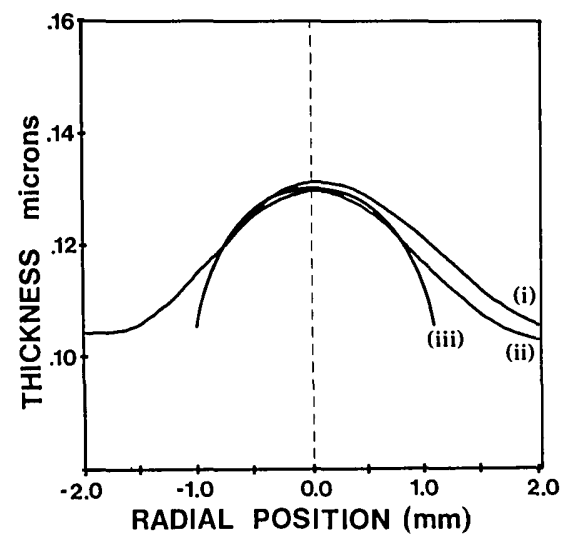

Fig. 5. Thickness profiles of lenslike waveguides showing $(i)$ profile uncompensated for the effects of gravity on structure symmetry, $(i i)$ profile compensated for effects of gravity, and (iii) ideal thickness profile which yields perfect periodic focusing with a focal length of $40.0 \mathrm{~mm}$.

are insufficient to drive away completely the solvents in the dip coating solution; thus pockets of solvent remain trapped and the resulting films are thicker with lower refractive indices. At higher temperatures, solvent evaporation is complete, and the resulting films are pure $\mathrm{SiO}_{2}: \mathrm{TiO}_{2}$. We have used baking temperatures of $500^{\circ} \mathrm{C}$. At this temperature, film shrinkage is almost complete after $15 \mathrm{~min}$, after which the refractive index slowly increases with increased baking time. One hour has been chosen as the optimum baking time at this temperature.

The microprocessor-controlled dipping arm is shown schematically in Fig. 4. The desired thickness profile is entered into the microprocessor, which has been previously programmed and calibrated to allow the calculation of the velocities required to achieve a desired profile. The microprocessor then controls the speed of the lifting arm via an 8-bit digital-to-analog converter, providing a series of digital signals which specify the duration and magnitude of each discrete speed interval. The D-A converter then provides a specific regulated voltage to the motor, whose lifting speed varies with the applied voltage. We have used lifting speeds which vary from $\sim 50$ to $250 \mathrm{~mm} / \mathrm{min}$ in $\sim 1-\mathrm{mm} / \mathrm{min}$ intervals and which are typically updated every $4 \mathrm{~ms}$. To fabricate the structure illustrated in Fig. 1(b), for example, requires 200 discrete lifting speeds varied over $12 \mathrm{~s}$.

Tapered optical waveguides have been produced with linear tapers and thickness changes of the order of $0.01 \mu \mathrm{m} / \mathrm{mm}$ along the waveguide. Thickness profiles were measured using a scanning ellipsometer technique.

The fabrication of symmetrical structures such as lenslike waveguides is complicated by the effects of gravity. Curve (i) in Fig. 5 shows an initial attempt to fabricate a region of varying thickness which will periodically focus a guided wave. The resulting asymmetry in the thickness profile is apparent. The effects of gravity were investigated by depositing tapered waveguides with identical lifting speed patterns but either increasing or decreasing in thickness. By comparing the taper angles of two such structures, and to some degree simply using a trial and error procedure, we were able to compensate for gravity as shown in curve (ii).

\section{Summary}

Thin film waveguide structures with varying thickness profiles have been fabricated using a microprocessor controlled dip coating procedure. Following standardization of the dip coating parameters, linear tapers and lenslike waveguides have been produced. Current work is on refining the profile control to allow the fabrication of thin film lenses for the focusing and collimating of guided waves. Experimental work describing the fabrication and properties of a solution deposited thin film lens will be presented in a future paper.

The authors wish to thank J.P. Danylyk for assisting with the dip coating and J. Ord and D. Becksteed for their help with the ellipsometry measurements. Thanks are also due to BDH Chemicals and E. Merck for supplying the Liquicoat solutions and technical information. This project is supported by the Natural Sciences and Engineering Research Council of Canada.

This work was presented at the 1987 Annual Meeting of the Optical Society of America in Rochester NY, 19-23 Oct. 1987.

\section{References}

1. J. H. Harris, R. Shubert, and J. N. Polky, "Beam Coupling to Films," J. Opt. Soc. Am. 60, 1009 (1970).

2. R. Ulrich and H. P. Weber, "Solution Deposited Thin Films as Passive and Active Light Guides," Appl. Opt. 11, 428 (1972).

3. P. P. Herrman and D. Wildmann, "Fabrication of Planar Dielectric Waveguides with High Optical Damage Threshold," IEEE J. Quantum Electron. QE-19, 1735 (1983).

4. W. Lukosz and K. Tienfenthaler, "Embossing Technique for Fabricating Integrated Components in Hard Inorganic Waveguide Materials," Opt. Lett. 8, 537 (1983).

5. K. Tienfenthaler, V. Briguet, E. Buser, M. Horisberger, and W. Lukosz, "Preparation of Planar Optical $\mathrm{SiO}_{2}-\mathrm{TiO}_{2}$ and $\mathrm{LiNbO}_{3}$ Waveguides with a Dip Coating Method and an Embossing Technique for Fabricating Grating Couplers and Channel Waveguides," Proc. Soc. Photo-Opt. Instrum. Eng. 401, 165 (1983).

6. K. Heuberger and W. Lukosz, "Embossing Technique for Fabricating Integrated Components in Hard Inorganic Waveguiding Materials," Appl. Opt. 25, 1499 (1986).

7. D. W. Hewak and J. W. Y. Lit, "Standardization and Control of a Dip Coating Procedure for Optical Thin Films Prepared from Solution," accepted for publication Can. J. Phys. special issue on Optics (1988). 